Table I. Partial molal heat capacities of amino acids at infinite dilution in various solvents at $25^{\circ}$.

\begin{tabular}{|c|c|c|c|c|c|c|c|}
\hline \multirow{2}{*}{\multicolumn{2}{|c|}{ Amino acida }} & \multicolumn{6}{|c|}{$C p_{2}{ }^{\circ}(\mathrm{cal} / \mathrm{mole} \cdot \mathrm{deg})$} \\
\hline & & $\mathrm{H}_{2} \mathrm{O}$ & $\mathrm{D}_{2} \mathrm{O}$ & $\begin{array}{c}10 \% \\
\text { Ethanol }\end{array}$ & $\begin{array}{c}6 M \\
\text { Urea }\end{array}$ & $\begin{array}{l}2 m \\
\mathrm{KI}\end{array}$ & $\begin{array}{c}2 m \\
\mathrm{NaCl}\end{array}$ \\
\hline \multicolumn{2}{|r|}{ Methionine } & $\begin{array}{l}84 \pm 5 \\
(0.22)\end{array}$ & $\begin{array}{l}56 \pm 5 \\
(0.14)\end{array}$ & $\begin{array}{l}63 \pm 3 \\
(0.10)\end{array}$ & $\begin{array}{l}64 \pm 10 \\
(0.19)\end{array}$ & $\begin{array}{l}61 \pm 10 \\
(0.14)\end{array}$ & $\begin{array}{r}126 \pm 6 \\
(0.14)\end{array}$ \\
\hline \multicolumn{2}{|r|}{ Norvaline } & $\begin{array}{l}84 \pm 5 \\
(0.30)\end{array}$ & $\begin{array}{l}68 \pm 5 \\
(0.35)\end{array}$ & $\cdots$ & $\cdots$ & $\cdots$ & $\ldots$ \\
\hline \multicolumn{2}{|r|}{ Glycine } & $\begin{array}{l}7.5 \pm 0.5^{b} \\
(3.33)\end{array}$ & $\begin{array}{l}15 \pm 3 \\
(1.45)\end{array}$ & $\begin{array}{l}27 \pm 2 \\
(1.76)\end{array}$ & $\begin{array}{l}35 \pm 3^{\circ} \\
(2.30)\end{array}$ & $\begin{array}{l}54 \pm 5 \\
(0.68)\end{array}$ & $\begin{array}{l}30 \pm 3 \\
(0.88)\end{array}$ \\
\hline \multicolumn{2}{|r|}{ Met-Gly } & $76 \pm 5.5$ & $41 \pm 10$ & $36 \pm 5$ & $29 \pm 13$ & $7 \pm 15$ & $96 \pm 9$ \\
\hline \multirow{2}{*}{\multicolumn{2}{|c|}{ Norval-Gly }} & $76 \pm 5.5$ & $53 \pm 8$ & .. & $\ldots$ & $\ldots$ & $\ldots$ \\
\hline & & 0.999 & 1.006 & 1.025 & 0.816 & 0.728 & 0.882 \\
\hline \multicolumn{4}{|c|}{$\begin{array}{l}\text { a The numbers in parentheses indicate the highest concentrations } \\
\text { (molality) at which measurements of } c \not \text { were made. } \\
{ }^{b} \text { C. A. Zittle and C. L. A. Schmidt, J. Biol. Chem. 108, } 161 \text { (1935) and }\end{array}$} & \multicolumn{4}{|c|}{$\begin{array}{l}\text { checked at Cornell University. } \\
\text { ' G. C. Kresheck and L. Benjamin, J. Phys. Chem. 68, 2476 (1964). } \\
\text { d Data in this row represent the specific heat of the solvent, } c p^{\circ} \text {. }\end{array}$} \\
\hline \multicolumn{4}{|c|}{$\begin{array}{l}\text { Table I. The observed difference in heat capacity in } \\
\mathrm{H}_{2} \mathrm{O} \text { between methionine and glycine (Met-Gly) and } \\
\text { between norvaline and glycine (Norval-Gly) give rise } \\
\text { to values for the nonpolar side chains of } 76 \mathrm{cal} / \mathrm{mole} \cdot \mathrm{deg} \\
\text { which are similar to that of } 85.5 \mathrm{cal} / \mathrm{mole} \cdot \mathrm{deg} \text { pre- } \\
\text { viously determined for Val-Gly. }{ }^{6} \mathrm{Also} \text {, the heat capacity } \\
\text { difference between Met-Gly in water and } 6 \mathrm{M} \text { urea is } \\
\text { consistent with the structure breaking effects of urea } \\
\text { observed previously for amino acid and dipeptide } \\
\text { solutions. } \\
\text { The main intent of this communication is to point out }\end{array}$} & \multicolumn{4}{|c|}{ 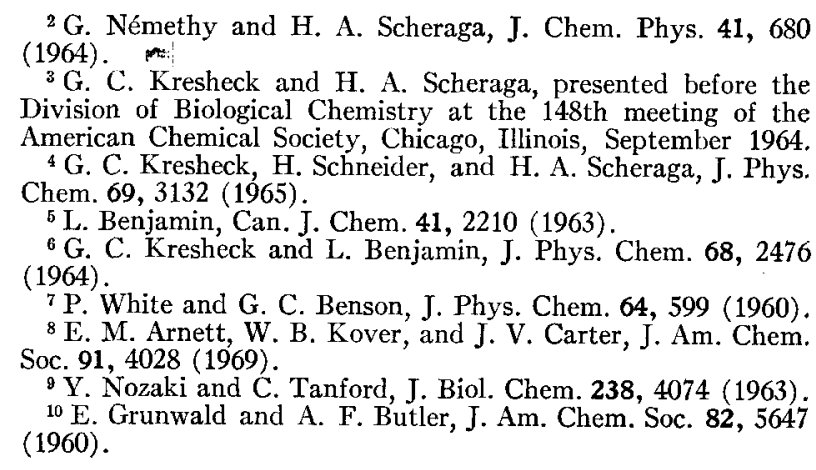 } \\
\hline
\end{tabular}
$\mathrm{H}_{2} \mathrm{O}$ and $\mathrm{D}_{2} \mathrm{O}$. Lower heat capacities were observed in each case with $\mathrm{D}_{2} \mathrm{O}$ solutions. Since it is possible to interpret an excess heat capacity in solutions with enhanced solvent structure, ${ }^{6,8}$ it may be concluded that the nonpolar side chains of methionine and norvaline promote less structure in $\mathrm{D}_{2} \mathrm{O}$ than in $\mathrm{H}_{2} \mathrm{O}$ in agreement with the results obtained by Lovett and Ben-Naim.

Although comparison of the heat capacity data in the other solvents with theory is not possible, it is interesting to note that the partial molal heat capacity associated with the nonpolar methionine side chain is less in $10 \%$ ethanol, $2 m \mathrm{KI}$, and $6 M$ urea than in water and larger in $2 m \mathrm{NaCl}$ than in water. Nonpolar amino acids have been shown to be more soluble than polar ones in $6 M$ urea and in $10 \%$ ethanol solutions than in water, ${ }^{9}$ and $\mathrm{KI}$ may be regarded as an electrolyte which tends to salt-in nonpolar molecules whereas $\mathrm{NaCl}$ tends to salt them out. ${ }^{10}$ Therefore, from the limited amount of heat capacity data available it appears as though there may be a useful correlation between salting-in and salting-out effects and partial molal heat capacity of solute molecules in aqueous solutions, excluding any specific interactions which may occur.

* The technical assistance of Mrs. E. Stimson and Mr. J. Vournakis is gratefully acknowledged.

1 R. A. Lovett and A. Ben-Naim, J. Chem. Phys. 51, 3108 (1969).

\section{Sum Rule for Interference Scattering from a Rigid Rotor*}

\author{
N. A. LuRIE \\ Department of Physics, University of Missouri, \\ Columbia, Missouri 65201 \\ AND \\ J. M. CARpenter
}

Department of Nuclear Engineering, The University of Michigan, Ann Arbor, Michigan 48105

(Received 10 December 1969)

Sum rules, or moment relations, have been frequently used in slow neutron scattering to provide a check on measured quantities ${ }^{1}$ and theoretically calculated expressions. ${ }^{2}$ They were first introduced in this context by Placzek. ${ }^{3}$ The importance of these relations has been emphasized by many authors, including de Gennes, ${ }^{2}$ Nelkin, ${ }^{4}$ and Schofield. ${ }^{5}$ We examine here a case which has not been worked out previously.

The $n$th frequency moment of the scattering law is defined by

$$
\left\langle\omega^{n}\right\rangle=\int_{-\infty}^{\infty} \omega^{n} S(\boldsymbol{\kappa}, \omega) d \omega,
$$


where the scattering law is written formally as

$$
\begin{aligned}
S(\boldsymbol{\kappa}, \omega) & =(2 \pi)^{-1} \int_{-\infty}^{\infty} d t \\
& \times \exp (i \omega t) \sum_{\mu \nu} a_{\mu \nu}{ }^{2}\left\langle\exp \left(i_{\boldsymbol{k}} \cdot \mathbf{r}_{\nu}\right) \exp \left[-i \mathbf{k} \cdot \mathbf{r}_{\mu}(t)\right]\right\rangle_{\boldsymbol{T}},
\end{aligned}
$$

and where $\hbar \omega$ and $\hbar$ represent the energy and momentum gained by the neutron. We are concerned here with the first frequency moment, $\left\langle\omega^{1}\right\rangle$, and for this purpose, have used Placzek's expression ${ }^{3}$ for the first moment,

$$
\left\langle\omega^{1}\right\rangle=\hbar^{-1} \sum_{\mu \nu} a_{\mu \nu}{ }^{2}\left\langle\exp \left(i_{\mathbf{k}} \cdot \mathbf{r}_{\boldsymbol{v}}\right)\left[\exp \left(-i \mathbf{k} \cdot \mathbf{r}_{\mu}\right), H\right]\right\rangle_{T}
$$

where $a_{\mu \nu}{ }^{2}$ is the product of the scattering lengths for the $\mu$ th and $\nu$ th nuclei, $\mathbf{r}_{\mu}$ is the position operator for the $\mu$ th nucleus, $H$ is the system Hamiltonian, and $\langle\cdots\rangle_{\boldsymbol{T}}$ represents a thermal average of expectation values of the enclosed operator. The sums on both $\mu$ and $\nu$ run over all nuclei of the system.

There are a number of systems of interest for which this expression can be evaluated without approximation. For systems described by Hamiltonians of the form

$$
H=\sum_{v=1}^{N} \frac{p_{v}^{2}}{2 M_{v}}+V\left(\mathbf{r}_{1}, \mathbf{r}_{2}, \cdots, \mathbf{r}_{N}\right)
$$

the moments up to order $n=4$ were obtained by Placzek $^{3}$ for direct scattering $(\nu=\mu)$, and up to $n=3$ for interference scattering $(\nu \neq \mu)$. The fourth order term was computed by de Gennes, ${ }^{2}$ in a classical approximation. It is found that the first frequency moment vanishes for interference scattering.

We consider here the case of the rigid diatomic molecule, which has been discussed previously by Kosaly and Solt. ${ }^{6}$ They examined the zeroth through third moments, but only for direct scattering. Not only was the exact problem considered, but also two approximate models (the Krieger-Nelkin ${ }^{7}$ and mass tensor ${ }^{8}$ ) which have been of interest in describing the scattering of slow neutrons by molecules. They were particularly interested in seeking improvements to those approximations.

For cases in which the interference scattering is important, it is necessary to evaluate the first moment for the interference part of the scattering law.

The Hamiltonian for a rigid rotating diatomic molecule with fixed center of mass is ${ }^{9}$

$$
H=L^{2} / 2 I,
$$

where $I$ is the principal moment of inertia and $L$ is the angular momentum operator. This is not of the form of Eq. (3). Using this Hamiltonian in Expression (2) and summing the exact expression for the expectation values, we have evaluated the first moment for the general case and obtained the result in a simple closed form

$$
\begin{aligned}
\left\langle\omega^{1}\right\rangle= & (-\hbar / I) \sum_{\mu \nu} a_{\mu \nu}{ }^{2} \kappa b_{\mu} \\
& \times\left\{\left(\kappa b_{\mu} / 3\right)\left[j_{0}(\kappa b)+j_{2}(\kappa b)\right]-j_{1}(\kappa b)\right\} \\
= & (-\hbar / I) \sum_{\mu \nu} a_{\mu \nu}{ }^{2} \kappa b_{\mu} j_{1}(\kappa b)\left[b_{\mu} / b-1\right]
\end{aligned}
$$

where $b_{\mu}$ is the equilibrium displacement of the $\mu$ th atom from the center of mass, and $b=b_{\nu}-b_{\mu}$, with $b=|\mathbf{b}|$, and where $j_{l}(x)$ is the spherical Bessel function of order $l$. For the special case of direct scattering this reduces to the well-known result

$$
\left\langle\omega^{1}\right\rangle=\frac{-\hbar \kappa^{2}}{3 I} \sum_{\nu=1}^{2} a_{\nu \nu}{ }^{2} b_{\nu}{ }^{2} .
$$

The interference contribution $(\nu \neq \mu)$ clearly does not vanish identically although it approaches an oscillating function of constant amplitude for large $\kappa$, and is then negligible compared to the direct terms. The corresponding terms vanish identically for Hamiltonians of the form of (3). The difference comes about because of the assumption of the rigid-rotor Hamiltonian, which is well justified to describe many realistic situations. This is the case when the internal vibration is of such high frequency that it has no effect on the measured cross section, except at very large energy transfers. The present result thus represents the first moment not only for calculations based on a rigid-rotor model, but also for measurements in cases where scattering with large energy transfer can be ignored.

We have checked our expression for the first moment (5) by comparing against the result of integrating the scattering law [the right-hand side of Eq. (1)] which had been computed according to the exact quantummechanical theory.10,11 This was done for two values of the momentum transfer, $3.5 \AA^{-1}$ and $6.3 \AA^{-1}$, for a heteronuclear diatomic molecule (hydrogen chloride) and a homonuclear diatomic molecule (nitrogen). In all cases the results agreed within the error limits of our graphical integration (about $3 \%$ ).

The authors express their gratitude to Professor G. C. Summerfield for valuable discussions.

* Work supported by the National Science Foundation.

1 P. D. Randolph, Phys. Letters 3, 162 (1963); Phys. Rev. 134, A1238 (1964); K. E. Larsson, in Thermal Neutron Scattering, edited by P. A. Egelstaff (Academic, London, 1965), p. 402.

2 P. G. de Gennes, Physica 25, 825 (1959).

${ }^{3}$ G. Placzek, Phys. Rev. 86, 377 (1952).

${ }^{4}$ M. Nelkin, Proceedings of the Symposium on Inelastic Scattering of Neutrons (IAEA, Vienna, 1961), p. 3.

5 P. Schofield, Ref. 4, p. 39.

${ }^{6}$ G. Kosaly and G. Solt, Phys. Letters 6, 51 (1963); 13, 223 (1964); Physica 32, 16 (1966).

${ }_{7}$ T. J. Krieger and M. S. Nelkin, Phys. Rev. 106, 290 (1957).

\& See for example, J. A. Janik and A. Kowalska, in Thermal Neutron Scattering, edited by P. A. Egelstaff (Academic, London, 1965), Chap. 9.

${ }^{9}$ Center-of-mass motion does not need to be included here since its effect in general can be treated exactly, and since it makes no contribution to the interference part of the first moment.

${ }_{10}$ A. Rahman, J. Nucl. Energy 13, 128 (1961).

11 N. A. Lurie, J. Chem. Phys. 46, 352 (1967). 